\title{
Ballistic transport properties in pristine/doped/pristine graphene junctions
}

\author{
J.S. Ardenghi ${ }^{\dagger}$, P. Bechthold ${ }^{\dagger}$, E. Gonzalez ${ }^{\dagger}$, P. Jasen ${ }^{\dagger}$ and A. Juan ${ }^{\dagger}$ \\ ${ }^{\dagger}$ IFISUR, Departamento de Física (UNS-CONICET) \\ Avenida Alem 1253, Bahía Blanca, Argentina
}

July 23,2018

\begin{abstract}
We investigate the ballistic electron transport in a monolayer graphene with configurational averaged impurities, located between two clean graphene leads. It is shown that the electron transmission are strongly dependent on the concentration of impurities and the incident energy. In turn, the conductance computed using the Landauer formalism shows a similar behavior to those found in experimental works as a function of the applied voltage for different concentrations of impurities in the limit of low temperatures. In the limit of zero bias voltage, the conductance shows a minimum value which reduces to zero for high concentration of impurites which disentangle graphene sublattices. These results can be very helpful for exploring the tunneling mechanism of electrons through doped thermodynamically stable graphene.
\end{abstract}

\section{Introduction}

Graphene, a new material with promising application possibilities and important fundamental physics aspects, is a two-dimensional allotrope of carbon which has become one of the most significant topics in solid state physics $([1,2,[3,[4,[5])$. The carbon atoms form a honey-comb lattice made of two interpenetrating triangular sublattices, $A$ and $B$. A special feature of the graphene band structure is the linear dispersion at the Dirac points which are dictated by the $\pi$ and $\pi^{\prime}$ bands that form conical valleys touching at the high symmetry points of the Brillouin zone [6. Electrons near these symmetry points behave as massless relativistic Dirac fermions with an effective Dirac-Weyl Hamiltonian [4.

The understanding of charge transport mechanism of pristine graphene/doped graphene is crucial for future applications in nanoelectronics. As clean graphene being a gapless semimetal, is useless for electronic development, therefore it is necessary to turn graphene from semimetallic to a gap semiconductor, which can be realized in several ways (with substrates 7 by confinement 8 and quantum dots [9]). In the other side, a quasigap in the vicinity of the Dirac point can be obtained in two dimensional system lattice sites with two different site energies and different probabilities [10. In this work we report a theoretical model to describe the transport mechanism in the ballistic regime at the interface of clean graphene and graphene with adatoms with arbitrary energies (positive values as donor and negative values as acceptor) placed on a site-like position. The impurities are randomized and averaged over its possible positions which transform the diffusive system in a ballistic one. In this sense, the system can be considered in thermodynamic equilibrium with a fixed number of impurity concentration. The diffusive character of systems with disorder can be study through Green function techniques (see [11, 12, 13]) and Kubo formalism, which allow to obtain the quantum corrections to the conductivity and other effects as weak antilocalization 11 For the purpose of this work, we will

\footnotetext{
*email: jsardenghi@gmail.com, fax number: +54-291-4595142

${ }^{1}$ Although for ideal graphene, the dynamics of the electrons produce the same shot noise as that found in classical diffusion (see [14).
} 
consider that the quantum mechanical coherence length is longer than the sample size $L$, in this case, the disorder of random impurities is transformed in a mass term in the Hamiltonian by the averaging procedure. Then by applying Landauer formalism ([15]) is enough to study the ballistic behavior of Bloch electrons through the sample 2 Graphene-based devices have not been fully investigated due to the complex processes required to achieve p- and n-doped semiconducting graphene. By chemical doping, graphene-based p-n junctions can be obtained ([17, [18]), but graphene retain its semimetallic character. In this work, from the impurity averaged tight-binding Hamiltonian in the long wavelength limit, is possible to obtain a Dirac equation with mass for the Bloch electrons. In this sense, a gap in the energy band near the Fermi energy is obtained. The tunnel junction studied is based on pristine graphene/doped graphene/pristine graphene (PG/DG/PG). The transport mechanism is highly dependent on the impurity concentrations and several predictions can be obtained through theoretical calculations to be applied to different metal/semiconductor junctions (see [19]).

The paper is organized as follows. In Section II, we introduce the tight-binding Hamiltonian with impurities and the averaging procedure. Then, the long-wavelength approximation is applied to obtain Dirac equation with mass. In Section III, Landauer formalism to the PG/DG/PG system is applied for low temperatures. The conductance as a function of the applied voltage is obtained. Minimum conductance is computed for zero voltage. Finally, an equation relating the length of the sample as a function of the concentration of impurities can be computed to obtain a transmission coefficient which is indepenent on the impurity concentration.

In the final section, the conclusions are presented. In appendix A, the coefficients of the Taylor expansion of the conductance as a function of the impurities are computed.

\section{Tight binding model with impurities}

The tight-binding Hamiltonian of graphene for nearest neighbors reads

$$
H_{0}=-t \sum_{\langle i, j\rangle, \sigma}\left(a_{i, \sigma}^{\dagger} b_{j, \sigma}+b_{i, \sigma}^{\dagger} a_{j, \sigma}\right)
$$

where $a_{i, \sigma}^{\dagger}\left(a_{i, \sigma}\right)$ creates (annihilates) an electron on site $\mathbf{r}_{\mathbf{i}}$ with spin $\sigma$, where $\sigma=\uparrow, \downarrow$ on sublattice $A$ and $b_{i, \sigma}^{\dagger}\left(b_{i, \sigma}\right)$ creates (annihilates) an electron on site $\mathbf{r}_{\mathbf{i}}$ with spin $\sigma$, on sublattice $B$ and $t$ is the nearest neighbor $\langle i, j\rangle$ hopping energy. Impurities can be included in the tight-binding description by the addition of a local energy term

$$
H_{i m p}=\sum_{i, \sigma}^{N_{i}}\left(V_{i} a_{i, \sigma}^{\dagger} a_{i, \sigma}+W_{i} b_{i+\delta, \sigma}^{\dagger} b_{i+\delta, \sigma}\right)
$$

where $V_{i}$ is a random potential at site $\mathbf{r}_{\mathbf{i}}$ and $W_{i}$ is a random potential at site $\mathbf{r}_{\mathbf{j}}$ and where $\delta=a(1,0,0)$. By introducing the Fourier transform of the annihilation and creation operators $a_{i, \sigma}$ and $b_{i, \sigma}$ :

$$
a_{i, \sigma}=\frac{1}{\sqrt{N}} \sum_{\mathbf{k}} e^{i \mathbf{k} \mathbf{r}_{\mathbf{i}}} a_{\mathbf{k}, \sigma} \quad b_{i, \sigma}=\frac{1}{\sqrt{N}} \sum_{\mathbf{k}} e^{i \mathbf{k r}_{\mathbf{i}}} b_{\mathbf{k}, \sigma}
$$

The Hamiltonian reads

$$
H=\sum_{\mathbf{k}, \sigma}\left[\phi(\mathbf{k}) a_{\mathbf{k}, \sigma}^{\dagger} b_{\mathbf{k}, \sigma}+\phi^{*}(\mathbf{k}) b_{\mathbf{k}, \sigma}^{\dagger} a_{\mathbf{k}, \sigma}\right]+\frac{1}{N} \sum_{\mathbf{k}, \mathbf{q}, \sigma}\left(V(\mathbf{q}) a_{\mathbf{k}, \sigma}^{\dagger} a_{\mathbf{k}+\mathbf{q}, \sigma}+W(\mathbf{q}) b_{\mathbf{k}, \sigma}^{\dagger} b_{\mathbf{k}+\mathbf{q}, \sigma}\right)
$$

where

$$
V(\mathbf{q})=\sum_{i}^{N_{i}} V_{i} e^{i \mathbf{q} \mathbf{r}_{\mathbf{i}}} \quad W(\mathbf{q})=\sum_{i}^{N_{i}} W_{i} e^{i \mathbf{q} \mathbf{r}_{\mathbf{i}}}
$$

\footnotetext{
${ }^{2}$ Other theoretical methods can be applied to obtain the effects of impurities in the electronic spectra of graphene (see [16]).
} 
A configurational averaging over the impurites can be applied over the Hamiltonian of last equation, that is, the lattice points where the impurities are located can be placed at random with different random configurities 3 We can sum over all the configurations of possible positions of impurities in the lattice. If there are $N_{i}$ impurities, then the configurational averaging can be computed as (see [13])

$$
\langle F\rangle=\frac{1}{A^{N_{i}}} \sum_{\mathbf{r}_{1}} \ldots \sum_{\mathbf{r}_{\mathbf{N}_{\mathbf{i}}}} F\left(\mathbf{r}_{1}, \ldots, \mathbf{r}_{\mathbf{N}_{\mathbf{i}}}\right)
$$

where $A$ is the area of graphene sheet. The configurational averaged Hamiltonian reads

$$
\langle H\rangle=\sum_{\mathbf{k}}\left[\phi(\mathbf{k}) a_{\mathbf{k}, \sigma}^{\dagger} b_{\mathbf{k}, \sigma}+\phi^{*}(\mathbf{k}) b_{\mathbf{k}, \sigma}^{\dagger} a_{\mathbf{k}, \sigma}+V a_{\mathbf{k}, \sigma}^{\dagger} a_{\mathbf{k}, \sigma}+W b_{\mathbf{k}, \sigma}^{\dagger} b_{\mathbf{k}, \sigma}\right]
$$

where

$$
V=\frac{1}{N} \sum_{i}^{N_{i}} V_{i} \quad W=\frac{1}{N} \sum_{i}^{N_{i}} W_{i}
$$

and

$$
|\phi(\mathbf{k})|=-t \sqrt{1+4 \cos ^{2}\left(\frac{\sqrt{3}}{2} k_{y} a\right)+4 \cos \left(\frac{3}{2} k_{x} a\right) \cos \left(\frac{\sqrt{3}}{2} k_{y} a\right)}
$$

and where we have used eq.(6). Hamiltonian of eq.(7) can be diagonalized and the spectrum reads

$$
E_{\lambda}(\mathbf{k})=\frac{V+W}{2}+\lambda \sqrt{\left(\frac{V-W}{2}\right)^{2}+|\phi(\mathbf{k})|^{2}}
$$

where $\lambda= \pm 1$, where the plus sign is for the conduction band and the minus sign for the valence band. To study the behavior of electrons at the Dirac point, we can expand the energy near the $K$ Dirac point $\left(\frac{2 \pi}{3 a}, \frac{2 \pi}{3 \sqrt{3} a}\right)$

$$
E_{\lambda}(\mathbf{p})=\lambda \sqrt{\frac{\gamma^{2}}{4}+\frac{3}{4} \frac{t^{2} a^{2}}{\hbar^{2}} p^{2}}
$$

where $\gamma=V-W$ and $\mathbf{p}=\hbar \mathbf{k}$ and where we the constant term $\frac{V+W}{2}$ has been absorbed into a redefinition of the energy. This low-energy description is valid as long as the characteristic energy is smaller than a cutoff $E_{C} \sim \frac{\hbar v_{F}}{a} \sim 2.6 \mathrm{eV}$ of the order of the inverse lattice spacing (see [20]).

The last Hamiltonian is the Hamiltonian of a massive Dirac fermions, where the mass and velocity reads

$$
m=\frac{2 \gamma \hbar^{2}}{3 t^{2} a^{2}}
$$

and

$$
v_{F}=\frac{\sqrt{3} t a}{2 \hbar}
$$

which is identical to the Fermi velocity of clean graphene. In this sense, graphene with impurities in the long wavelength approximation can be considered as fermions satisfying the Dirac Hamiltonian with mass given by eq.(12) and velocity given by eq.(13). The mass of electrons is proportional to $\gamma$, which depends on the impurity potentials. In the case that $V_{i}=V_{0}$ and $W_{i}=W_{0}, V=n_{i} V_{0}$ and $W=$ $n_{i} W_{0}$, where $n_{i}=N_{i} / N$ is the impurity concentration. The mass term due to the impurities can be interpreted as if graphene is altered by a periodic potential which originates an effective mass for the propagation of electrons. This mass term is not a diffusive term in the Hamiltonian, then it cannot

\footnotetext{
${ }^{3}$ In general, configurational averaging is applied over the Green function (see [13]). The average restore the translation symmetry of the system, but transforms the original system of non-interacting electrons to a correlated one. Configurational averaging applied directly over the Hamiltonian restore traslation invariance and the impurities appears as an effective mass term. In this sense, the average over the Hamiltonian disables the disorder introduced in eq. (4).
} 
gives information about many-body effects for electrons in graphene with impurities. Nevertheless, impurities are still there in the Hamiltonian as a mass term and can give a detailed description for ballistic transport phenomena, provided that the quantum mechanical coherence length is longer than the sample size $L$. Even more, graphene with random impurities is no longer a disorder system when the average is applied to the Hamiltonian, but inertial effects appears in electrons.

\subsection{Dirac equation}

By apply the quantization procedure to the energy of eq.(10) we can obtain the Dirac equation

$$
E \psi=\left(v_{F} \vec{\sigma} \cdot \vec{p}+\sigma_{z} m v_{F}^{2}\right) \psi
$$

where $\vec{\sigma}$ are the Pauli matrices $\sigma_{x}$ and $\sigma_{y}$. The solution of the Dirac equation reads

$$
\psi_{\lambda}(\mathbf{r})=\frac{1}{\sqrt{2}}\left[\begin{array}{c}
\sqrt{1+\frac{\lambda m v_{F}^{2}}{\sqrt{m^{2} v_{F}^{4}+v_{F}^{2} p^{2}}}} \\
\lambda e^{i \phi_{p}} \sqrt{1-\frac{\lambda m v_{F}^{2}}{\sqrt{m^{2} v_{F}^{4}+v_{F}^{2} p^{2}}}}
\end{array}\right] e^{\frac{i}{\hbar} \mathbf{p} \cdot \mathbf{r}}
$$

where

$$
\phi_{p}=\operatorname{arctg}\left(\frac{p_{y}}{p_{x}}\right)
$$

In the limit of no impurities, $m=0$ the spinor of eq.(15) is identical to the one of clean graphene as it is expected In the limit of high concentration of impurities $m \rightarrow \infty$ the spinor decouples the pseudospin

$$
\lim _{m \rightarrow \infty} \psi_{+}(r)=\left[\begin{array}{l}
1 \\
0
\end{array}\right] e^{\frac{i}{\hbar} \mathbf{p} \cdot \mathbf{r}}
$$

and

$$
\lim _{m \rightarrow \infty} \psi_{-}(r)=\left[\begin{array}{l}
0 \\
1
\end{array}\right] e^{\frac{i}{\hbar} \mathbf{p} \cdot \mathbf{r}}
$$

which implies that the impurities break the symmetry of $A$ and $B$ sublattice.

As we said before, graphene with random impurities has been transformed to graphene with a periodic potential that introduces an effective mass which is proportional to the impurity concentration. In this sense, the wave function of eq.(15) encodes the probability amplitude of finding a Bloch electron in the whole sample of lentgh $L$. This wave function do not contains information about a possible localization of the electron due to the interference effects of the impurities. Nevertheless, if the sample of length $L$ is placed near a sample of clean graphene, the wave functions must match in the boundary, which introduces several restrictions to the energies involved for the electron propagation. The fact that electrons in graphene with a configurational averaging of impurities applied to the Hamiltonian introduces an effective mass proportional to the impurity concentration, implies that the restrictions to the energies involved will be sensitive to the impurities through the mass term. From this result, it is possible to obtain a detailed description of the transport phenomena in the ballistic regime where the wave function is not localized.

\section{Tunneling transport through a potential barrier}

Consider the following two-dimensional model where doped graphene is placed between two reservoirs with different chemical potentials $\mu_{L}$ and $\mu_{R}$ connected through pristine graphene leads (see Figure 1). By matching the solution of Dirac equation for $x<0$ with $m=0$ with the solution of Dirac

\footnotetext{
${ }^{4}$ The limit $m=0$ can be obtained with $\gamma=0$ which implies that both impurity potentials $V$ and $W$ can be identical.
} 


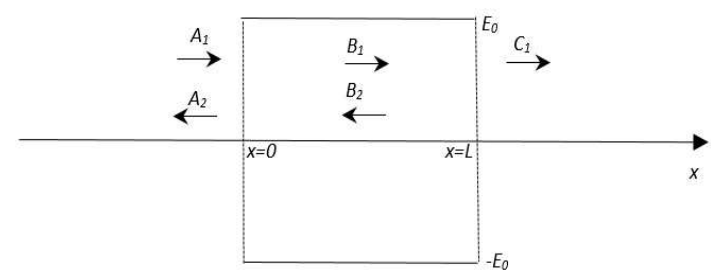

Figure 1: Schematic illustration of the tunnel junction composed of pristine graphene in the left and right leads and graphene with impurities in the scattering region.

equation for $0<x<L$ (see eq.(15) ) and with the solution of Dirac equation for $x>L$ we obtain for the transmission as a function of the incident energy 5

$$
T_{n}(E)=\frac{16\left|\eta_{n}\right|^{2}}{\left|e^{-i \xi_{n} L}\left(\eta_{n}+1\right)^{2}-e^{i \xi_{n} L}\left(\eta_{n}-1\right)^{2}\right|^{2}}
$$

where

$$
\eta_{n}(E)=\frac{\hbar v_{F}\left(\xi_{n}-i \alpha_{n}\right)}{E-E_{0}}
$$

and

$$
\xi_{n}(E)=\frac{1}{\hbar v_{F}} \sqrt{E^{2}-E_{0}^{2}-\hbar^{2} v_{F}^{2} \alpha_{n}^{2}}
$$

where $E_{0}=m v_{F}^{2}$ and $\alpha_{n}=\frac{n \pi}{D}$, where $D$ is the width of the sample ribbon. The allowed energy are in the range $-E_{C}<E<E_{C}$. For the $n=0$ mode, the transmission coefficient reads

$$
T_{0}(E)=\frac{16 \eta_{0}^{2}}{16 \eta_{0}^{2}+4\left(\eta_{0}^{2}-1\right)^{2} \sin ^{2}\left(\xi_{0} L\right)}
$$

where the maximum are located when $\xi_{0} L=m \pi$, for $m=\in \mathbb{Z}$

$$
E_{\max }=\sqrt{\left(\frac{\hbar v_{F} m \pi}{L}\right)^{2}+E_{0}^{2}}
$$

The first mode $n=0$ is independent of the width of the ribbon $D$ due to the the factor $\alpha_{n}=\frac{n \pi}{D}$, which implies that the conductance and transmission are valid for any value of $D 6$ The Landauer formula for the total current flowing from the left to right lead in the $n=0$ mode is given by

$$
I_{T}=I_{L \rightarrow R}-I_{R \rightarrow L}=g_{s} \frac{2|e|}{\hbar} \int_{-E_{C}}^{E_{C}} d E T_{0}(E)\left(f_{L}\left(E, \mu_{L}\right)-f_{R}\left(E, \mu_{R}\right)\right)
$$

where $g_{s}=2$ is the spin degeneracy factor and where $f(E, \mu)$ is the Fermi-Dirac distribution

$$
f_{L(R)}\left(E, \mu_{L(R)}\right)=\frac{1}{1+\exp \left[\beta\left(E-\mu_{L(R)}\right)\right]}
$$

\footnotetext{
${ }^{5}$ The $y$ direction has a length $D$ which introduces a quantization of the $p_{y}$ component of the momentum.

${ }^{6}$ This could be appropiate for some experimental purpose, since it is not necessary to consider one of the sample dimension.
} 


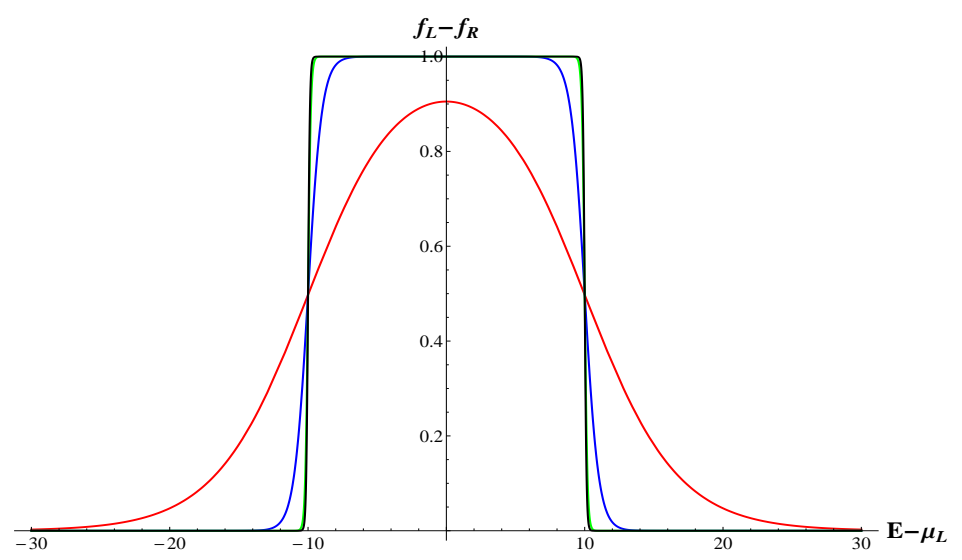

Figure 2: Gate function for different temperatures (red line $\beta=0.3$, blue line $\beta=2$, green line $\beta=10$ and black line $\left.\beta=15,|e| V_{S D}=20\right)$.

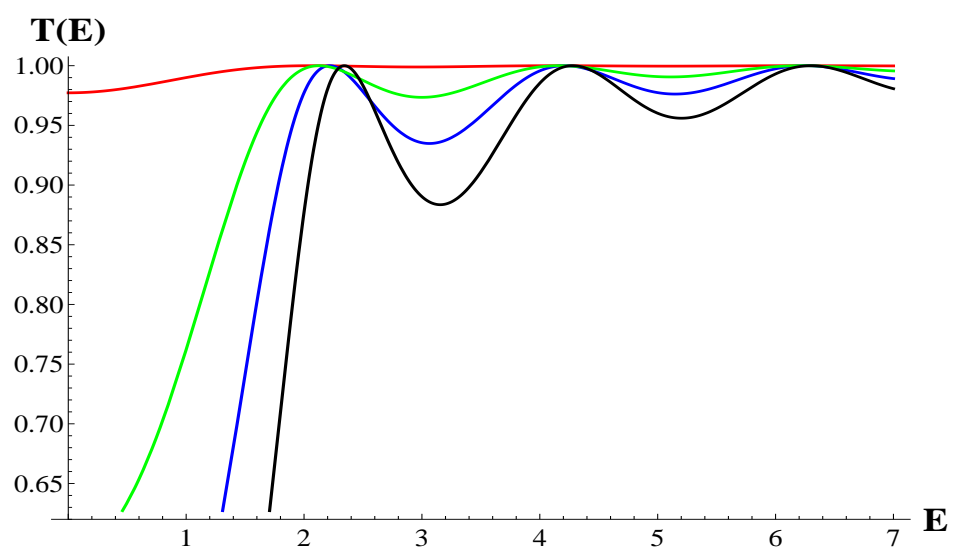

Figure 3: Transimission as a function of incident energy for different concentration of impurities (red line $E_{0}=0.1$, green line $E_{0}=0.4$, blue line $E_{0}=1.25$, black line $E_{0}=1.7$ ).

where $\beta=1 / k_{B} T$. If we assume that the chemical potentials are related as $\mu_{R}=\mu_{L}+|e| V_{S D}$ where $V_{S D}$ is the source-drain voltage difference between the leads and we shift the energy as $E \rightarrow E+\frac{e V_{S D}}{2}$, then the difference of Fermi distributions can be written as a gate function

$$
g(\beta)=f_{L}\left(E, \mu_{L}\right)-f_{R}\left(E, \mu_{R}\right)=-\frac{\sinh \left(\frac{\beta e V_{S D}}{2}\right)}{\cosh \left(\frac{\beta e V_{S D}}{2}\right)+\cosh \left(\beta\left(E-\mu_{L}\right)\right)}
$$

which behaves at low temperatures as $f_{L}\left(E, \mu_{L}\right)-f_{R}\left(E, \mu_{R}\right) \sim 1$ between $\mu_{L}-\frac{e V_{S D}}{2}$ and $\mu_{L}+\frac{e V_{S D}}{2}$ and zero in the remaining energy values (see 2). As $\beta$ goes down, the gate function goes to zero by relaxing the behavior of rectangular function. For $\beta \rightarrow \infty$, the integral of eq.(24) reads

$$
\int_{-E_{C}}^{E_{C}} d E T_{0}(E)\left(f_{L}\left(E, \mu_{L}\right)-f_{R}\left(E, \mu_{R}\right)\right)=\int_{\mu_{L}}^{\mu_{L}+e V_{S D}} T_{0}(x) d x
$$

In last equation, the limits of the integral are located between $\mu_{L}$ and $\mu_{L}+|e| V_{S D}$ in the case that $E_{C}>\mu_{L}+|e| V_{S D}$. In the other side $E_{C}<\mu_{L}+|e| V_{S D}$, the upper limit will be $E_{C}$. The conductance $G=I_{T} / V_{S D}$ can be computed up to order $E_{0}^{6}$ due to the complexity of the integrand of last equation. 


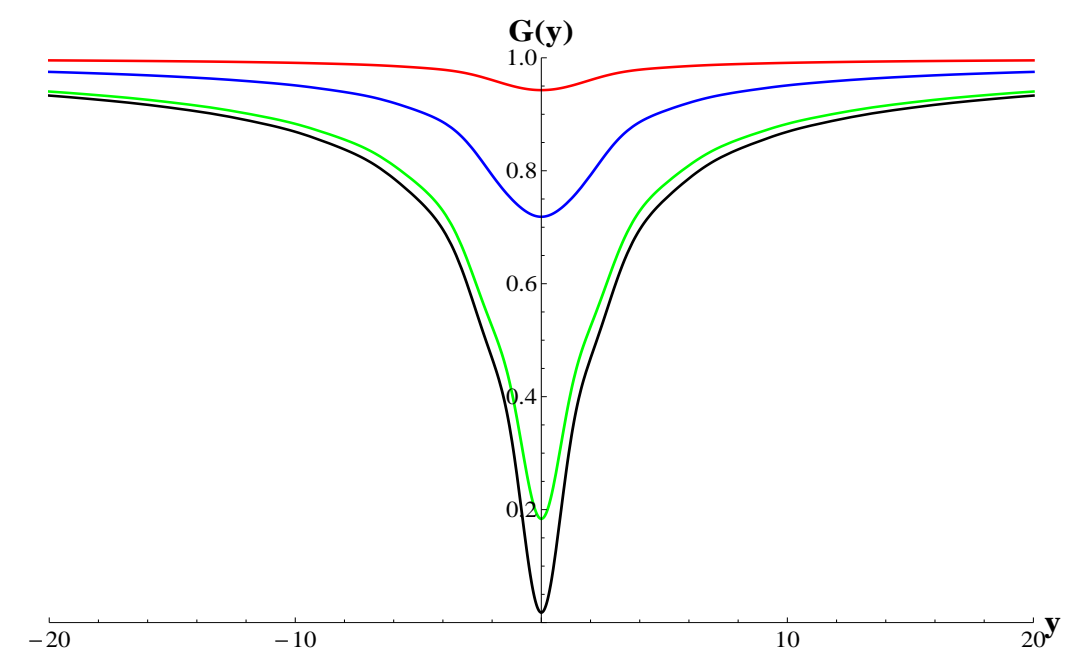

Figure 4: Conductance as a function of the source-drain voltage for different concentration of impurities (red line $E_{0}=0.2$, blue line $E_{0}=0.4$, green line $E_{0}=0.8$, black line $E_{0}=0.84$ ). The unit of the $x$-axis is dimensionless.

The limit $\mu_{L} \rightarrow 0$ can be taken without loss of generality, then

$$
G=\frac{4|e|}{\hbar V_{g}}\left(f_{0}\left(V_{S D}\right)+f_{2}\left(V_{S D}\right) E_{0}^{2}+f_{4}\left(V_{S D}\right) E_{0}^{4}+f_{6}\left(V_{S D}\right) E_{0}^{6}+O\left(E_{0}^{8}\right)\right)
$$

where the coefficients $f_{j}\left(V_{S D}\right)$ are shown in Appendix A. In figure 4, the contributions of the different orders in $E_{0}$ can be obtained for the conductance $G$ as a function of a dimensionless variable $y=$ $\frac{L|e| V_{S D}}{\hbar v_{F}}$, which is proportional to $V_{S D}$. The limit $V_{S D} \rightarrow 0$ can be taken in $G$ and reads

$$
\lim _{V_{S D} \rightarrow 0} G=\frac{4|e|^{2}}{\hbar} T_{0}(0)=\frac{4|e|^{2}}{\hbar} \frac{1}{1+\sinh ^{2}(x)}
$$

where $x$ is a dimensionless variable which reads $x=\frac{L E_{0}}{\hbar v_{F}}$. Last result is the correction to the minimum conductivity without source-drain voltage. Several theoretical explanations can be found in the literature related to the chiral nature of low energy excitations (see [21, [22, [12]). In figure 3, the electron transmission as a function of the electron energy for different concentration of impurities is shown 7 As it is expected, the transmission probability is suppressed for energy values less than $E_{0}$, which can be ascribed to the enhancement of the reflection. For low impurity concentration $\left(E_{0}<0.2\right.$, red line in figure 3) transmission probability do not show transmission gap for incident energies below $E_{0}$, similar to the results found in [23. In order to see what extent the transmission properties are reflected in measurable quantities which involve averaging over the impurities in the ballistic regime, we plot the conductance as a function of the source-drain voltage for different concentration of impurities (see figure 4). As it is expected, the conductance decrease when the source-drain voltage goes to zero. The value of the minimum of the conductance is lower for higher values of impurity concentrations, in corcondance with experimental reports (see [24, fig. 2a, 25], fig.8, 26], fig. 4), where the energy introduced by the impurities plays the rol of $k T$. In turn, the variation of the conductance with respect the source-drain voltage can reflect the effects found in [27] (fig. 4). The peculiar minimum value for $V_{S D}=0$ is different than zero as it is expected for graphene. In eq.(29), the minimum of the conductance is computed (see figure 6). For no impurities $E_{0}=0$, the conductance is $G=\frac{4|e|^{2}}{\hbar}$, although the density of states has no charge carriers at the Fermi energy. For high values of impurities,

\footnotetext{
${ }^{7}$ The energy unit of figures 3 and 7 are $\mathrm{eV}$.
} 


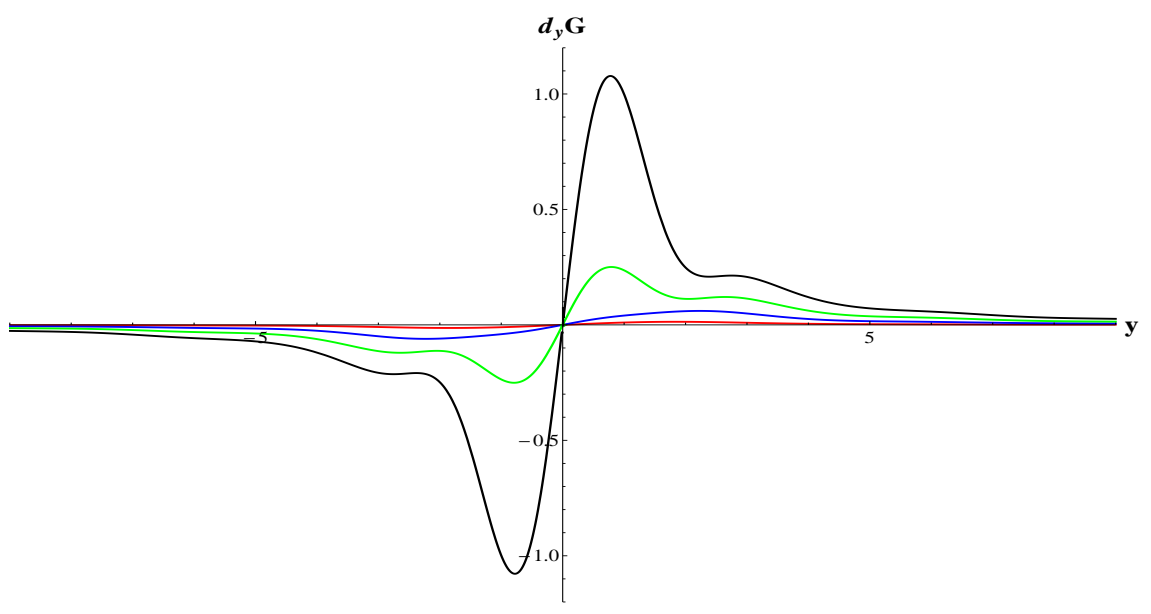

Figure 5: $\frac{d G}{d y}$ as a function of the source-drain voltage (the values of $E_{0}$ correspond to figure 4).

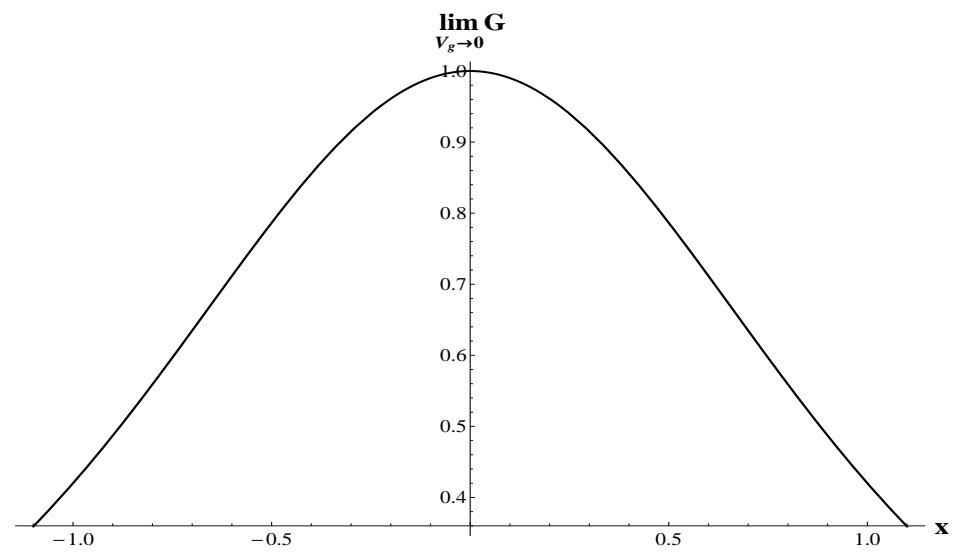

Figure 6: Minimum conductance as a function of concentration of impurities. 
the minimum conductivity goes to zero, which correspond to the disentangling of sublattices $A$ and $B$ (see eq.(17) and eq.(18) ) and no Zitterbewegung effect.

Another relevant point is wether the effective mass $m$ of eq. (12) can be introduced in the DrudeSommerfield model for diffusive conductivity. For a simple inspection, the conductivity $\sigma$ is proportional to $n_{i}^{-1}$ which is a typical behavior of solids (see 28]). In the other side, using that the relaxation time $\tau=l / v_{F}$ and $l \sim 1 / \sqrt{n_{i}}$ (see [29], [30]), the conductivity is proportional to $\beta=n / n_{i}^{3 / 2}$ which is a dimensionless parameter that separates the diffusive $(\beta<<1)$ from the ballistic regime $(\beta>>1)$ (see [31]). In this sense, the effective mass description for the diffusive regime in graphene is highly sensitive in the relation between charge carriers and impurity concentration.

With the purpose of obtaining a transmission coefficient with no no dependence in the concentration of impurities, PG/DG/PG junctions can be realized by traslating the $E_{0}$ dependence into $L$. For this, the following renormalization equation can be obtained

$$
\frac{d T_{0}}{d E_{0}}=\frac{\partial T_{0}}{\partial E_{0}}+\frac{\partial T_{0}}{\partial L} \frac{d L}{d E_{0}}=0
$$

which is a non-linear first order differential equation

$$
\frac{d L}{d E_{0}}=-\frac{\hbar v_{F} E^{2}}{E_{0}\left(E^{2}-E_{0}^{2}\right)^{3 / 2}} \tan \left(\frac{L \sqrt{E^{2}-E_{0}^{2}}}{\hbar v_{F}}\right)+\frac{E_{0} L}{E^{2}-E_{0}^{2}}
$$

Transmission gap is below $E_{0}$, which implies that for practical purposes, the r.h.s. of last equation can be expanded in Taylor series around $E=E_{0}$

$$
\frac{d L}{d E_{0}}=-\frac{L}{E_{0}}-\frac{L^{3} E_{0}}{3 \hbar^{2} v_{F}^{2}}
$$

which is valid at order $O\left(E^{0}\right)$. The solution of last differential equation reads

$$
L\left(E_{0}\right)= \pm \frac{L(1) \sqrt{3} \hbar v_{F}}{\sqrt{3 \hbar^{2} v_{F}^{2} E_{0}^{2}+2 L^{2}(1) E_{0}^{2} \ln \left(E_{0}\right)}}
$$

where $L(1)$ is the length of the sample of doped graphene when $E_{0}=\frac{V-W}{2}=1$. Last equation is valid only for incident energy $E \sim E_{0}$. For impurity concentrations below $E_{0}=e^{-\frac{3}{2}\left(\frac{\hbar v_{F}}{L(1)}\right)^{2}}$, real solution for $L$ does not exist. In fig. 7, the reference value is $L(1)=10^{-9} m$ and $L(1)=10^{-6} m$. For values $E_{0}<1$, the length of the doped graphene sample in both cases increase considerably. These interesting phenomena can provide an important reference to the design of various electronic devices based on graphene with energy gap, but where the impurities only introduces a minimum threshold for the electron transmission coefficient. In turn, the renormalization methods that relates the length of the sample with the impurity concentration can give some insight of how the diffusive and ballistic regimes are related by applying Shot noise measurements (see [14]), where the conductance variations are measured as a function of the sample length of doped and clean graphene.

Finally, is interesting to note that the averaging procedure introduces a gap in the energy band as it is occur in the Haldane model (see 32. and for a more accurate version see [33). The Haldane model depends on an inversion symmetry breaking on-site energy $M$ for the sublattice $A$ and $-M$ on sublattice $B$ and a complex hopping amplitude between next-nearest neighbor due to the Peierls subtitution, which is obtained by applying a staggered magnetic field, which is positive near the center of each hexagon and negative near the edges which results in a zero net flux in the hexagon. In our model, random impurities are introduced as adsorbates that change the on-site energies where they are located. In the model introduced in this work, the particle-hole symmetry is not destroyed because we do not take into account next-nearest neighbor bonds. The crucial point is that the random impurities breaks the translational symmetry and the inversion symmetry. With the averaging procedure, the translational symmetry is restored, but the inversion symmetry is not. In fact, the mass term in our 


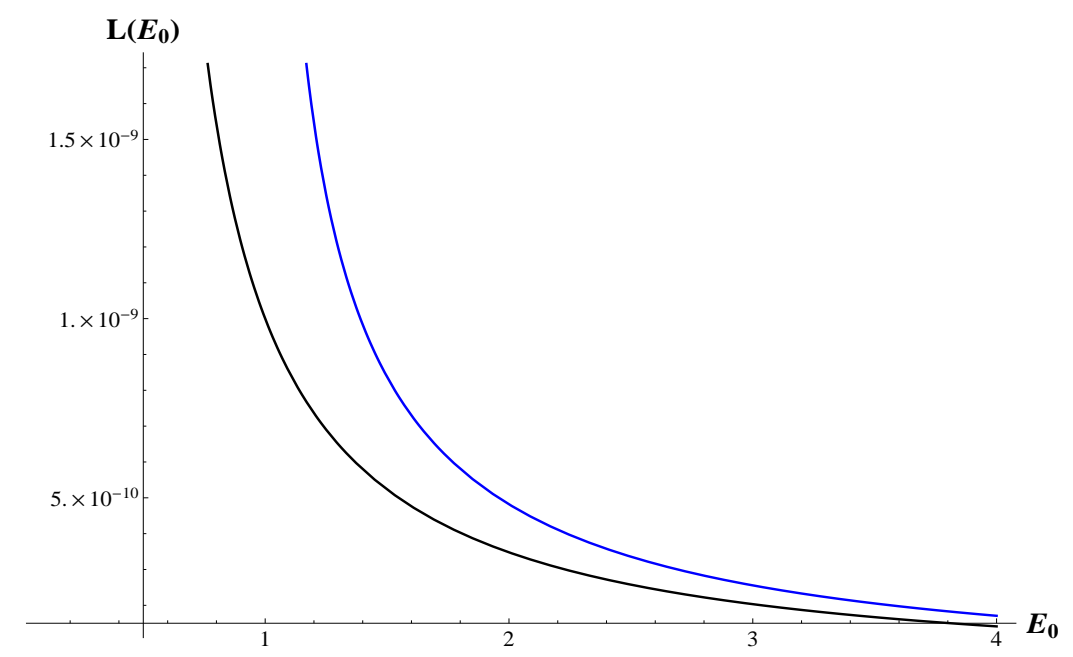

Figure 7: Length of the doped graphene sample as a function of the impurity concentration. Black line for $L(1)=10^{-9} \mathrm{~m}$ and blue line for $L(1)=10^{-6} \mathrm{~m}$.

model depends on $V-W=\frac{1}{N} \sum_{i=1}^{N_{i}}\left(V_{i}-W_{i}\right)$, where $V_{i}$ is the impurity located in sublattice $A$ and $W_{i}$ is the impurity located in sublattice $B$. If both values are the same, $V_{i}=W_{i}$, then the mass term is zero, which implies that Bloch electrons cannot distinguish between the upper side of the sample with respect the other side, that is, the inversion symmetry is restored and no gap is obtained 8 In this sense, our model is a particular case of the Haldane model, where $t_{2}=0$ and $-W_{i}=V_{i}=M$.

\section{Conclusion}

In this paper we have studied the transport mechanism of pristine/doped/pristine graphene junction in the ballistic regime. The conductance as a function of the impurity concentration has been computed using Landauer formalism with the application of the averaging procedure on the impurity positions over the Hamiltonian. Minimum conductivity is obtained exactly for low temperatures. A renormalization equation was obtained for the sample length and the impurity concentration with the purpose to obtain a transmission coefficient that do not depends on the impurities. This result can be of importance for the manufacturing of Schootky junctions with gapped doped graphene. Finally, the model introduced in Section II is related to the Haldane model, finding that the band gap obtained is due to breaking inversion symmetry introduced by the position-averaged impurities.

\section{Appendix}

The coefficients of the Taylor expansion of the transmission of eq. (19) as a function of $E_{0}$ up to order $O\left(E_{0}^{6}\right)$ reads

$$
\begin{gathered}
f_{0}(y)=\frac{\gamma y}{L} \\
f_{2}(y)=\frac{L}{\gamma}\left(\frac{1}{y} \sin ^{2}(y)-S i(2 y)\right)
\end{gathered}
$$

\footnotetext{
${ }^{8}$ The condition $V_{i}=W_{i}$ is not the unique condition for no gap in the energy bands. Because we are taking an averaging over the possible locations of the impurities, no matter if an on-site energy $V_{i}$ is not equal to $W_{i}$ provided that there is another on-site energy that is $W_{j}=-V_{i}$.
} 


$$
\begin{aligned}
f_{4}(y)= & \frac{L^{3}}{24 \gamma^{3} y^{3}}\left(-12 y^{2} \cos (2 y)+\left(8 y^{2}-1\right) \cos (4 y)+1\right. \\
& \left.\left.-6 y \sin (2 y)+2 y \sin (4 y)-24 y^{3} \operatorname{Si}(2 y)+32 y^{3} \operatorname{Si}(4 y)\right)\right)
\end{aligned}
$$

and

$$
\begin{gathered}
f_{6}(y)=\frac{L^{5}}{960 \gamma^{5} y^{5}}\left(12+2\left(3+29 y^{2}-58 y^{4}\right) \cos (2 y)-\right. \\
4\left(3+16 y^{2}-128 y^{4}\right) \cos (4 y)-6\left(1-3 y^{2}+54 y^{4}\right) \cos (6 y) \\
-y\left(33+58 y^{2}\right) \sin (2 y)+16 y\left(-3+8 y^{2}\right) \sin (4 y)+\left(9 y-54 y^{3}\right) \sin (6 y) \\
\left.-8 y^{5}(29 \operatorname{Si}(2 y)-256 \operatorname{Si}(4 y)+243 \operatorname{Si}(6 y))\right)
\end{gathered}
$$

where $\gamma=\hbar v_{F}$ and $y=\frac{L|e| V_{S D}}{\gamma}, S i(x)$ is the sine integral function.

\section{References}

[1] K. S. Novoselov, A. K. Geim, S. V. Morozov, D. Jiang, M. I. Katsnelson, I. V. Grigorieva, S. V. Dubonos and A. A. Firsov, Nature, 438, 197 (2005).

[2] A.K. Geim and K. S. Novoselov, Nature Materials, 6, 183 (2007).

[3] Y. B. Zhang, Y.W. Tan, H. L. Stormer and P. Kim, Nature, 438, 201 (2005).

[4] A. H. Castro Neto, F. Guinea, N. M. R. Peres, K. S. Novoselov and A. K. Geim, Rev. Mod. Phys., 81, 109 (2009).

[5] M. O. Goerbig, Rev. Mod. Phys., 83, 4 (2011).

[6] J. McClure, Phys. Rev., 104, 666 (1956).

[7] S.Y. Zhou, G.H. Gweon, A.V. Fedorov, P.N. First, W.A. de Heer, D.H. Lee, F. Guinea, A.H.C. Neto, A. Lanzara, Nature Mater., 6, 770 (2007).

[8] M.Y. Han, B. Ozyilmaz, Y. Zhang, P. Kim, Phys. Rev. Lett., 98, 206805 (2007).

[9] L.A. Ponomarenko, F. Schedin, M.I. Katsnelson, R. Yang, E.W. Hill, K.S. Novoselov, A.K. Geim, Science 320, 356 (2008).

[10] Yu.V. Skrypnyk, V.M. Loktev, Phys. Rev. B 73, 241402 (2006).

[11] V. Janis, J. Kolorenc, and V. Spicka, Eur. Phys. J. B 35, 77-91 (2003).

[12] K. Ziegler, Phys. Rev. B 75, 233407 (2007).

[13] J. Rammer, Quantum transport theory (Perseus books, Reading, Massachusetts, 1998).

[14] M. F. Borunda, H. Hennig, and E. J. Heller, Phys. Rev. B, 88, 125415 (2013).

[15] M. Di Ventra, Electronic Transport in Nanoscale Systems (Cambridge University Press, 2008).

[16] A. Feher, S. Feodosyev, I. Gospodarev, O. Kotlyar, K. Kravchenko, E. Manzhelii, E. Syrkin, Superlattices and Microstructures, 53, 55-62 (2013).

[17] J. R. Williams, L. DiCarlo, and C. M. Marcus, Science 317, 638 (2007).

[18] T. Lohmann, K. von Klitzing, and J. H. Smet, Nano Lett. 9, 1973 (2009). 
[19] C. Bai, J. Wang, Y. Yang, Superlattices and Microstructures, 43, 151-157 (2011).

[20] N. M. Peres, F. Guinea and H. Castro Neto, Phys. Rev. B, 73, 125411 (2006).

[21] M. I. Katsnelson, Eur. Phys. J. B 51, 157-160 (2006).

[22] M. Trushin and J. Schliemann, Phys. Rev. Lett., 99, 216602 (2007).

[23] J. Lu, B. Xu, H. Liu, Y. Wanga, W. Zheng, Superlattices and Microstructures 60 217-223 (2013).

[24] Yanbin An, Ashkan Behnam, Eric Pop, and Ant Ural, Appl. Phys. Lett., 102, 013110 (2013).

[25] H. Zhong, K. Xu, Z. Liu, G. Xu, L. Shi, Y. Fan, J. Wang, G. Ren, and H. Yang, Jour. Appl. Phys., 115, 013701, (2014).

[26] M. Mohammed, Z. Li, J. Cui, and T. Chen, Nanoscale Research Letters, 7:302 (2012).

[27] S. Rajput, M.X. Chen, Y. Liu, Y.Y. Li, M. Weinert and L. Li, Nature Communications, 4: 2752 (2013).

[28] Gerald. D. Mahan, Many-Particle Physics (Plenum Press, New York, 1990).

[29] T. Stauber, N. M. R. Peres, and F. Guinea, Phys. Rev. B, 76, 205423 (2007).

[30] J. Horng, C. Chen, B. Geng, C. Girit, Y. Zhang, Z. Hao, H. A. Bechtel, M. Martin, A. Zettl, M. F. Crommie, Y. R. Shen, and F. Wang, Phys. Rev. B, 83, 165113 (2011).

[31] M. M. Fogler, D. S. Novikov, L. I. Glazman, and B. I. Shklovskii, Phys. Rev. B, 77, 075420, (2008).

[32] F. D. M. Haldane, Phys. Rev. Lett., 61, 2015, (1988).

[33] D. Sticlet and F. Piéchon, Phys. Rev. B, 87, 115402, (2013). 